\title{
P○LYPUBLIE Polytechnique Montréal
}

Effectiveness of braces designed using computer-aided design and

Titre: manufacturing (CAD/CAM) and finite element simulation compared

Title: to CAD/CAM only for the conservative treatment of adolescent idiopathic scoliosis: a prospective randomized controlled trial

Auteurs: Nikita Cobetto, Carl-Éric Aubin, Stefan Parent, Julien Clin, Soraya

Authors: Barchi, Isabelle Turgeon, \& Hubert Labelle

Date: 2016

Type: Article de revue / Article

Cobetto, N., Aubin, C.-É., Parent, S., Clin, J., Barchi, S., Turgeon, I., \& Labelle, $\bar{H}$.

(2016). Effectiveness of braces designed using computer-aided design and

Référence: manufacturing (CAD/CAM) and finite element simulation compared to CAD/CAM

Citation: only for the conservative treatment of adolescent idiopathic scoliosis: a

prospective randomized controlled trial. European Spine Journal, 25 (10), 3056-

3064. https://doi.org/10.1007/s00586-016-4434-3

\section{Document en libre accès dans PolyPublie}

Open Access document in PolyPublie

URL de PolyPublie:

PolyPublie URL:

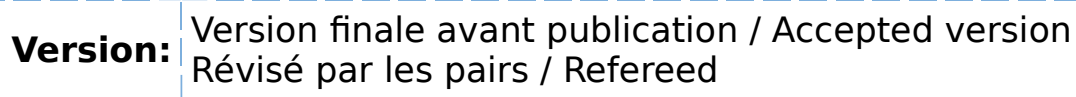

Conditions d'utilisation:
Terms of Use:

Document publié chez l'éditeur officiel

Document issued by the official publisher

Titre de la revue: Journal Title:

Maison d'édition:
Publisher:

URL officiel: Official URL:

Mention légale: This is a post-peer-review, pre-copyedit version of an article published in European Legal notice: $\begin{aligned} & \text { Spine Journal (vol. 25, no. 10). The final authenticated version is available online at: } \\ & \text { https://doi.org/10.1007/s00586-016-4434-3 }\end{aligned}$ 
Title: Effectiveness of Braces Designed Using Computer Aided Design and Manufacturing (CAD/CAM) and Finite Element Simulation Compared to CAD/CAM Only for the Conservative Treatment of Adolescent Idiopathic Scoliosis: a Prospective Randomized Controlled Trial.

\section{Authors:}

Cobetto Nikita ${ }^{1,2}$, Aubin Carl-Éric ${ }^{1,2,3}$, Parent Stefan ${ }^{2,3}$, Clin Julien ${ }^{1,2}$, Barchi Soraya ${ }^{2}$, Turgeon Isabelle ${ }^{2}$, Labelle Hubert ${ }^{2,3}$

1. Polytechnique Montréal

Department of Mechanical Engineering

P.O. Box 6079, Downtown Station

Montreal (Quebec), H3C 3A7, CANADA

2. Research Center, Sainte-Justine University Hospital Center

3175, Cote Sainte-Catherine Road

Montreal (Quebec), H3T 1C5, CANADA

3. Université de Montréal

Faculty of Medecine

P.O. Box 6128, Downtown Station

Montreal (Quebec), H3C 3J7, CANADA

Address for notification, correspondence and reprints:

Hubert Labelle, M.D.

Full Professor

CHU Sainte-Justine and Université de Montréal Motion Sciences Research Chair

Université de Montréal, Faculty of Medecine Surgery Departement

P.O. Box 6128, Downtown Station, Montreal (Quebec), H3C 3J7 CANADA

E-mail : hubert.labelle@recherche-ste-justine.qc.ca

Phone: 1 (514) 345-4931 ext. 5928; Fax: 1 (514) 345-4755 


\begin{abstract}
Purpose:

Clinical assessment of immediate in-brace effect of braces designed using CAD/CAM and FEM vs. only $\mathrm{CAD} / \mathrm{CAM}$ for conservative treatment of AIS, using a randomised blinded and controlled study design.
\end{abstract}

\title{
Methods:
}

Forty AIS patients were prospectively recruited and randomized into two groups. For 19 patients (control group), the brace was designed using a scan of patient's torso and a conventional $\mathrm{CAD} / \mathrm{CAM}$ approach (CtrlBrace). For the 21 other patients (test group), the brace was additionally designed using Finite Element Modeling (FEM) and 3D reconstructions of spine, rib cage and pelvis (NewBrace). The NewBrace design was simulated and iteratively optimized to maximize the correction and minimize the contact surface and material.

\section{Results:}

Both groups had comparable age, sex, weight, height, curve type and severity. Scoliosis Research Society standardized criteria for bracing were followed. Average Cobb angle prior to bracing was $27^{\circ}$ and $28^{\circ}$ for main thoracic (MT) and lumbar (L) curves respectively for the control group, while it was $33^{\circ}$ and $28^{\circ}$ for the test group. CtrlBraces reduced MT and L curves by $8^{\circ}(29 \%)$ and $10^{\circ}$ $(40 \%)$ respectively, compared to $14^{\circ}(43 \%)$ and $13^{\circ}(46 \%)$ for NewBraces, which were simulated with a difference inferior to $5^{\circ}$. NewBraces were $50 \%$ thinner and had $20 \%$ less covering surface than CtrlBraces.

\section{Conclusion:}

Braces designed with $\mathrm{CAD} / \mathrm{CAM}$ and 3D FEM simulation were more efficient and lighter than standard CAD/CAM TLSO's at first immediate in-brace evaluation. These results suggest that long-term effect of bracing in AIS may be improved using this new platform for brace fabrication.

\section{Keywords:}

Computer-aided design/computer-aided manufacturing, Scoliosis, Thoraco-lumbo-sacral orthosis, Finite element modeling (FEM), RCT 


\section{Manuscript}

\section{Introduction}

Adolescent Idiopathic Scoliosis (AIS) is a threedimensional (3D) deformity of the spine and rib cage. For Cobb angles between $20^{\circ}-40^{\circ}$, a conservative brace treatment is generally prescribed $[1,2]$. Recent studies demonstrated bracing as an effective treatment to prevent curve progression[36]. A correlation was found between immediate in-brace correction and brace treatment's long-term effectiveness[7-8]. Brace treatment effectiveness also relies on timing with adolescent growth spurt, spine flexibility and patient compliance to treatment[9-12].

Traditional brace fabrication method for rigid thoraco-lumbo-sacral orthoses (TLSO) involves taking a mold of the patient later filled with plaster to create a positive cast which is then modified by adding/removing plaster at different areas. This manual technique requires time, material consumption and presents a low accuracy[13]. Computer-aided design and manufacturing systems (CAD/CAM) have proven to be as effective compared to plaster-cast methods[14-16]. The trunk surface acquisition can be realized using a surface topography system[17] or optical scanners $[17,18]$. The CAD/CAM method is also cost and time effective as it increases productivity (2.5 times) compared with plaster-cast methods and allows patient data storing for future references[13]. However, none of these techniques allow testing brace efficacy before the patient wears it.

Finite element models (FEM) have been used to analyze brace biomechanics[19-24]. A detailed and realistic FEM was developed to simulate brace treatment[15,16,23,25]. Combined to a $\mathrm{CAD} / \mathrm{CAM}$ system, FEM allows brace correction simulation and computation of pressures applied on a patient's torso. Iterative improvement of the brace design and its biomechanical efficiency assessment can be made before brace fabrication. Recently, a clinical evaluation using the FEM was done on a group of 22 scoliotic patients[15,16] to improve functional design of braces. Each participating patient received two braces to compare brace effectiveness. The first brace was fabricated using the CAD/CAM-FEM method while the second brace was fabricated using the conventional plaster-cast technique. These studies showed the feasibility of using CAD/CAM and FEM to fabricate braces as efficient as standard TLSO like the Boston brace system. The predicted correction results were similar to that of the real fabricated brace and comfort parameters were integrated to brace design without compromising biomechanical efficiency. However, these 
feasibility studies are limited by the fact that patients were not randomized and by the use of the plaster-cast technique as the control group.

The purpose of this study was to perform a randomized and controlled trial (RCT) to assess the braces effectiveness designed using CAD/CAM-FEM vs. only CAD/CAM for the conservative treatment of AIS.

\section{Methods}

Experimental study design

40 patients were consecutively recruited on a voluntary basis over a 2 years period. All participants were diagnosed with an AIS, had an immature skeleton presenting a Risser sign 0-2 and were prescribed a full-time TLSO [26]. Patients with a curve magnitude between $20^{\circ}$ to $45^{\circ}$ were included in the study to take into account the intra-observer measurement variability in the measurement of the Cobb angle. The study was approved by our institutional ethical committee and each participant and their parents gave a written consent.

Patients were assigned to control and test groups using a simple randomization technique. Randomization sequence was generated by a randomization table: a simple block randomization list with a block size of 4 was prepared by a biostatistician not involved in recruitment and followup of the patients. In this study, the caregivers were blinded but not the orthotist. The 19 patients from control group received a TLSO fabricated using a scan of their torso and the CAD/CAM approach (CtrlBrace). The 21 patients from the test group received a TLSO also designed using the $\mathrm{CAD} / \mathrm{CAM}$ approach but additionally simulated using a FEM built from each patient's torso and personalized 3D reconstruction (spine, rib cage and pelvis), and computationally improved for correction (NewBrace)(Protocol for test group). For all patients, the brace was prescribed during their visit at the clinic and calibrated bi-planar postero-anterior (PA) and lateral (LAT) radiographs were taken using a low-dose digital radiography system (EOS ${ }^{\mathrm{TM}}$, EOS imaging, Paris, France). All patients had their brace designed by two orthotists having more than 10 years of experience with TLSO and 2 years of experience with CAD/CAM technology. The following radiological indices were measured: main thoracic (MT) and lumbar (L) Cobb angles, kyphosis and lordosis angles. Angle measurement was done using the semi-automatic Fuji Synapse system (Fuji Synapse System, Fujifilm holdings, Tokyo, Japan) and was realized by two experimented operators. Angle 
measurement accuracy using this system is inferior to $5^{\circ}$. Kyphosis was measured using two different methods: maximal kyphosis using the most inclined vertebrae and constrained kyphosis using vertebrae T4-T12. Lordosis was measured using vertebrae L1-S1. A device recording brace wear time using in-brace temperature measurement was incorporated to all braces to evaluate patient compliance (iButton, Boston Brace, USA).

\section{Protocol for the Control Group (CtrlBrace)}

Patient's external torso geometry was obtained using a surface topography system (3-dimensional Capturor, Creaform inc, Levis, Canada)[17]. Orthotists used the Rodin4D CAD/CAM system (Rodin4D, Groupe Lagarrigue, Bordeaux, France) to design the CtrlBrace geometry. Using the software's interactive tools, modifications of the CtrlBrace design were made to add/remove material in order to introduce pressure and relief areas. Tools to apply corrective translations on brace sections and symmetrize its geometry were also used. Braces were then fabricated using a numerically controlled carver (Model C, Rodin 4D, Groupe Lagarrigue, Bordeaux, France) linked to the $\mathrm{CAD} / \mathrm{CAM}$ system. According to the $\mathrm{CAD}$ model, a polyurethane foam bloc was carved and brace shell thermoforming was done using a heated copolymer sheet. The fabricated brace was trimmed and adjusted by the orthotist and brace effectiveness was assessed using simultaneous PA and LAT in-brace radiographs. The brace covering surface was measured using the CATIA CAD/CAM software (CATIA, Dassault Sytemes, Vélizy-Villacoublay, France) by importing the STL file of the brace design and by computing the area of the brace shell. Brace shell thickness and corrective pads thickness were measured by the orthotist following brace adjustment using a caliper tool.

\section{Protocol for the Test Group (NewBrace)}

Using the calibrated PA and LAT radiographs, the 3D reconstruction of the patient's spine, ribcage and pelvis was done[26]. Patient's external torso geometry was obtained using the same surface topography system as with the control group[17]. Radio-opaque markers visible on X-rays and trunk surface were a priori positioned on anatomical points of the patient's torso and were used to register the skeleton reconstruction and the external torso geometry. With a previously validated method, the trunk's overall registered geometry was used to create a personalized FEM using Ansys 14.5 software package (Ansys Inc., Canonsburg, PA, USA)[8,23]. The FEM includes thoracic/lumbar vertebrae, intervertebral discs, ribs, sternum, costal cartilages, ligaments, abdominal cavity and soft external tissues[19-25](Figure 1). Mechanical properties for anatomical 
structures were taken from published data obtained on typical human cadaveric spine segments [22,23,28,29].

Orthotists used the same CAD/CAM software as for CtrlBraces to design NewBraces. However, the brace design thereby obtained was then simulated using the FEM to assess its effectiveness. A brace FEM using polyethylene mechanical properties was created[24]. A surface-to-surface contact interface was made to model friction and force transfer from the brace shell to the patient's trunk surface[30]. The orthotist had to select sets of nodes on the brace FEM to represent strap fixation localization and had to virtually position the brace on the patient's FEM. The brace installation was then automatically simulated. During all simulation steps, the pelvis was fixed in space and the first thoracic vertebra (T1) was allowed to rotate and translate longitudinally. For a given simulation, the correction was assessed using post-processed Cobb angles, lordosis and kyphosis, as well as computed pressures applied on the torso and space between patient's skin and brace shell (brace fitting)(Figure 2).

Following brace simulation results, it was possible to modify the brace design to improve brace correction. The brace design was iteratively modified in the CAD/CAM software by varying the corrective pad localization and depth. Other design parameters like trim lines, relief zones, side of trochanteric pad location and openings on the brace were also modified and simulated to improve brace design. Braces were iteratively designed and simulated. Their performance was computationally assessed by the orthotist to maximize the correction using post-processed indices. The criterion for correction maximization was to incrementally accentuate pad depth by $5 \mathrm{~mm}$ until simulated spinal correction stayed stable even with the corrective region depth increasing $\left(2^{\circ} \mathrm{Cobb}\right.$ angle). The pad depth was however limited by the corresponding pressure applied on the torso to not exceed a $35 \mathrm{KPa}$ value, a threshold previously established by Cobetto et al. to respect patient comfort in braces[18]. Patient's comfort was optimized by controlling pressures applied on the torso and minimizing contact surfaces between the brace and the patient's skin. The numerical process required an average of 3 iterations per patient (minimum 2, maximum 6).

The optimal NewBrace was then fabricated using the same numerical controlled carver and thermoforming process. The NewBrace was trimmed by the orthotist and brace effectiveness was assessed using simultaneous PA and LAT radiographs. Brace covering surface and brace thickness was measured using the same method as for CtrlBraces. 
$\underline{\text { Statistical analysis }}$

Statistical data analysis were performed using STATISTICA 10.0 software package (Statistica, StatSoft Inc., Tulsa, OK, USA). To verify that both groups were statistically comparable, a paired t-test (95\% significance level) was apply to compare age, sex, weight, height curve type and curve severity between both groups. A statistical analysis was also realized using a Mann-Whitney test ( $95 \%$ significance level) to analyze if there was a significant difference for in-brace correction (MT and L Cobb angles) and in-brace kyphosis and lordosis angles between both groups. To justify the use of parametric (paired t-test) and non-parametric (Mann-Whitney test) statistical tests, a ShapiroWilk's test for normality was realized on each group.

\section{Results}

Forty patients aged between 10-16 years were consecutively recruited. Both groups had comparable age, sex, weight, height, skeletal maturity, curve type and severity (Table 1) and presented a normal distribution. The correction indices (MT/L Cobb angles, maximal/constrained kyphosis and lordosis) were measured on radiographs taken prior to brace treatment during the patient's first visit and on the in-brace radiographs (Figure 3). For control group, average Cobb angle prior to bracing was $27^{\circ}(\mathrm{MT})$ and $26^{\circ}(\mathrm{L})$. For test group, average Cobb angle prior to bracing was $33^{\circ}(\mathrm{MT})$ and $28^{\circ}(\mathrm{L})$. For both groups, maximal and constrained kyphosis were $25^{\circ}$ and $33^{\circ}$ respectively and average lordosis was $53^{\circ}$ prior to bracing. CtrlBrace reduced MT and L Cobb angles by $8^{\circ}(29 \%)$ and $10^{\circ}(40 \%)$ respectively. NewBrace reduced MT and L Cobb angles by $14^{\circ}(43 \%)$ and $13^{\circ}$ $(46 \%)$ which were simulated with a difference inferior to $5^{\circ}$ (Table 2). NewBrace correction was found to be statistically significantly greater than CtrlBrace with a $p$-value $=0.02(p<0.05)$ for the MT Cobb angle correction. For the L Cobb angle correction, no significant difference was found. For CtrlBraces, maximal and constrained in-brace kyphosis were reduced to $27^{\circ}$ and $19^{\circ}$ respectively compared to NewBraces for which maximal and constrained in-brace kyphosis were maintained at $30^{\circ}$ and $23^{\circ}$ respectively. In-brace lordosis was reduced to $45^{\circ}$ for both groups (Table 2). No significant differences were found for the in-brace maximal/constraint kyphosis and lordosis angles between both groups. When analyzing normality of both groups using the Shapiro-Wilk's test, results showed that distribution for NewBrace MT curve correction and CtrlBrace L curve correction was not normal (Table 2), justifying the use of the Mann-Whitney statistical test to compare in-brace results between both groups. When analyzing the simulated corrective pressures applied by the brace on patient's torso, highest pressures were located on thoracic and lumbar 
regions with maximal values between $25-35 \mathrm{KPa}$. Simulated pressures at axillary and trochanter extension regions were lower with maximal values between 20-25 KPa. The average brace wear time was 15 hours/day for both groups (min $8 \mathrm{hrs}$, max $22 \mathrm{hrs}$ ). Newbraces had an average of 20\% less covering surface than CtrlBraces. The thermoplastic sheet thickness used for brace shell was the same for both groups $(4 \mathrm{~mm})$. As for the pads added in CtrlBraces, the thickness was $13 \mathrm{~mm}$. For NewBraces, no foam pad (liner) was necessary. Because Foam pads were recovering on average $34 \%$ of the brace area, NewBraces were on average $50 \%$ thinner than CrtlBraces (Figure $3)$.

\section{Discussion}

Recent evidence has demonstrated that $\mathrm{CAD} / \mathrm{CAM}$ is as effective in fabricating braces for AIS compared to traditional plaster-cast method[14], while being more cost and time effective. Two preliminary studies[15-16] have suggested that adding a FEM to CAD/CAM may also improve brace efficacy. However, it remained to be demonstrated that the addition of a more complex FEM technology offers any advantage over CAD/CAM alone. The present study indicates that a clinically and statistically significantly greater immediate in-brace thoracic Cobb angle correction and similar (slightly greater) lumbar curve correction can be obtained with the addition of FEM to $\mathrm{CAD} / \mathrm{CAM}$, with the additional advantages of $50 \%$ thinner braces with $20 \%$ less covering body surface. Patient comfort was not evaluated in this study but it would be possible to hypothesize that these design parameters could improve the treatment's comfort and patient's quality of life. It remains to be shown in a future study. The average brace wear time was 15 hours/day for both groups which is comparable to previous studies demonstrating the variability of brace wear time in adolescent population[11,12] presenting average brace wear compliance from $47 \%$ [33] to $91 \%$ [34] of brace wear prescription. Considering the results of studies showing a significant positive association between brace wear time and treatment's success [35] and showing a significant increase of treatment's success for a brace wear time of more than 18 hours per day [36], evaluating patient's compliance as a factor influencing the long-term outcome of brace would be part of a future study.

Since a correlation has been reported between immediate in-brace correction and brace treatment long-term effectiveness[7-8], these results suggest that the use of a FEM simulation platform may also improve the long-term TLSO treatment efficacy. Using this platform, it is possible to simulate/test different brace designs and better define the treatment plan. It allows orthotists to 
simulate and predict in-brace correction for different pad configurations as well as for different trim lines and relief zones adjustment to optimize brace design. The use of the simulation platform allowed orthotists to analyze the pressures applied on patient's trunk and the contact surface between brace and patient's skin, in order to avoid excessive pressures and adjust the openings and relief zones on the brace. This has resulted in braces averaging $20 \%$ less covering surface and $50 \%$ thinner when fabricated with the CAD/CAM-FEM. In addition, pads were added for CtrlBraces during brace fitting to adjust the brace design. This step was not necessary for NewBraces, indicating that the simulation tool can be useful to rationalize and improve the brace design. This simulation platform can also be used to study the biomechanics of other types of braces, as it was realized in a previous study for the Providence and Charleston night-time braces [37, 40]. It could also be used to study or improve the brace design of other brace approaches like the Chêneau brace, an orthosis also used to treat scoliotic thoraco-lumbar curves and presenting possible spinal correction in the sagittal and the transverse planes [38-39].

In sagittal plane, TLSO's fabricated with traditional plaster-cast method have been criticized as causing hypokyphosis, a potentially harmful side-effect as hypokyphosis is already present in most AIS subjects and considered an important part of the deformity. It is reassuring to document that kyphosis was slightly less reduced with NewBraces compared to CtrlBraces, although not statistically significant. We believe that having access to spinal 3D reconstructions with CAD/CAM techniques allows orthotists to better visualize and address this hypokyphotic component, and possibly improve brace design with more proper $3 \mathrm{D}$ fitting of the brace.

The main strength of this study is the RCT design, which confirms and supports previous feasibility studies with CAD/CAM and FEM simulations for brace design in AIS. There are, however, limitations to this trial. First, only immediate in-brace effect was measured. Even if a correlation has been found between immediate in-brace correction and brace treatment long-term effectiveness[7,22], measuring only immediate correction may be insufficient. Nevertheless, these encouraging results indicate that a more time consuming and expensive RCT study of long-term effects in a larger cohort appears warranted. Long-term results will allow to evaluate patient's compliance to treatment and patient's flexibility as factors influencing brace outcomes. The second main limitation is that addition of FEM to CAD/CAM techniques is more time consuming and adds another level of complexity to brace fabrication. In the configuration tested, the simulation process adds 20-30 minutes to the design process. However, because the brace was optimized, time needed 
for the fitting was reduced (approximately $30 \mathrm{~min}$ ). Time calculation could still be further reduced to favor an even more efficient clinical use by orthotists.

\section{Conclusions}

Braces designed with $\mathrm{CAD} / \mathrm{CAM}$ and FEM simulation are more efficient at correcting thoracic curves at first immediate in-brace evaluation, with the advantages of being lighter than standard CAD/CAM TLSO's and requiring less body surface coverage. These results suggest that long-term effect of bracing in AIS may be improved by the use of this new platform for brace fabrication, and indicate that a study of long-term effects in a larger cohort appears warranted.

\section{Acknowledgements}

Project funded by the Natural Sciences and Engineering Research Council of Canada (RGPIN 239148-11) and the Canadian Institutes of Health Research (MOP-119455). Special thanks to Marie-Chantal Bolduc and Benoit Bissonnette from Orthèse-Prothèse Rive-Sud who contributed to the design and fabrication of the braces, and delivery to the patients.

Trial registration: NCT02285621

\section{Compliance with Ethical Standards}

\section{Conflict of Interest}

Research and development contract was obtained with Groupe Lagarrigue to develop and transfer a license of the simulation platform. Money was given to the university and the contract was not directly related to the presented RCT study. The RCT study presented in this paper was funded by a peer-reviewed grant from the Canadian Institutes of Health Research. The participating orthotists from Orthèse-Prothèse Rive-Sud received nothing of value to realize this study. 


\section{Ethical approval}

All procedures performed in this study involving human participants were in accordance with the ethical standards of the institutional ethical research committee and with the 1964 Helsinki declaration and its later amendments or comparable ethical standards.

\section{$\underline{\text { Informed consent }}$}

Informed consent was obtained from all individual participants included in the study and their parents.

\section{Reference list:}

1. Nachemson AL and Peterson LE (1995) Effectiveness of treatment with brace in girls who have adolescent idiopathic scoliosis. A prospective, controlled study based on data from the Brace Study of the Scoliosis Research Society. The Journal of Bone and Joint Surgery 77:815-822.

2. Trobisch p, Suess O, Schwab F (2010) Idiopathic scoliosis. Deutsches Ärzteblatt International 107(49):875-883.

3. Castro F (2003) Adolescent idiopathic scoliosis, bracing, and the Hueter-Volkmann principle. Spine 3:182-185.

4. Negrini S, Atanasio S, Fusco C, Zaina F (2009) Effectiveness of complete conservative treatment for adolescent idiopathic scoliosis (bracing and exercises) based on SOSORT management criteria: results according to the SRS criteria for bracing studies-SOSORT Award 2009 Winner. Scoliosis 4:19.

5. Negrini S, Aulisa AG, Aulisa L, et al. (2012) 2011 SOSORT guidelines: Orthopaedic and Rehabilitation treatment of idiopathic scoliosis during growth. Scoliosis 7:1-35.

6. Weinstein SL, Dolan LA, Wright JG, Dobbs MB (2013) Effects of Bracing in Adolescents with Idiopathic Scoliosis. The New England Journal of Medicine 369(16):1512-1521

7. Landauer F, Wimmer C, Behensky H (2003) Estimating the final outcome of brace treatment for idiopathic thoracic scoliosis at 6-month follow-up. Pediatric rehabilitation 6:201-207.

8. Clin J, Aubin CE, Sangole A, Labelle H, Parent S (2010) Correlation between immediate in-brace correction and biomechanical effectiveness of brace treatment in adolescent idiopathic scoliosis. Spine 35(18):1706-1713. 
9. Nault M, Parent S, Phan P, Roy-Beaudry M, Labelle H, Rivard M (2010) A modified Risser grading system predicts the curve acceleration phase of female adolescent idiopathic scoliosis. The Journal of bone and joint surgery American volume 92:1073-1081.

10. Lusini M, Donzelli S, Minnella S, Zaina F, Negrini S (2010) Brace treatment is effective in idiopathic scoliosis over $45^{\circ}$ : an observational prospective cohort controlled study. Spine Journal 14(9):1951-1956

11. Brox JI, Lange JE, Gunderson RB, Steen H (2012) Good brace compliance reduced curve progression and surgical rates in patients with idiopathic scoliosis. European Spine Journal 21:1957-1963

12. Aulisa GO, Giordano M, Falciglia F et al. (2014) Correlation between compliance and brace treatment in juvenile and adolescent idiopathic scoliosis: SOSORT 2014 award winner. Scoliosis 9:6

13. Wong MS (2011) Computer-aided design and computer-aided manufacture (CAD/CAM) system for construction of spinal orthosis for patients with adolescent idiopathic scoliosis. Physiother Theory Pract. 27(1):74-79

14. Wong MS (2005) A comparison of treatment effectiveness between the CAD/CAM method and the manual method for managing adolescent idiopathic scoliosis. Prosthet Orthot Int. 29(1):105-111.

15. Desbiens-Blais F, Clin J, Parent S, Labelle H, Aubin CE (2012) New Brace Design Combining CAD/CAM and Biomechanical Simulation for the Treatment of Adolescent Idiopathic Scoliosis. Clinical biomechanics 27:999-1005.

16. Cobetto N, Aubin CE, Clin J, Le May S, Desbiens-Blais F, Labelle H, Parent S (2014) Braces Optimized with Computer-Assisted Design and Simulations Are Lighter, Comfortable and More Efficient than plaster-casted braces for the treatment of Adolescent Idiopathic scoliosis. Spine Deformity 2(4): 276-284

17. Pazos V, Cheriet F, Dansereau J, Ronsky J, Zernicke RF, Labelle H (2007) Reliability of trunk shape measurements based on 3-D surface reconstructions. European Spine Journal 16:1882-1891.

18. Raux S, Kohler R, Garin C, Cunin V, Abelin-Genevois K (2014) Tridimensinal trunk surface acquisition for brace manufacturing in idiopathic scoliosis. European Spine Journal 4:S419-23. 
19. Aubin C, Descrimes JL, Dansereau J, et al. (1995) Geometrical modeling of the spine and the thorax for the biomechanical analysis of scoliotic deformities using the finite element method. Annales de chirurgie 49:749-761.

20. Gignac D, Aubin CE, Dansereau J, Labelle H (2000) Optimization method for 3D bracing correction of scoliosis using a finite element model. European Spine Journal 9:185-190.

21. Wynarsky GT, Schultz AB (1991) Optimization of skeletal configuration: studies of scoliosis correction biomechanics. J Biomech. 24(8):721-732.

22. Perie D, Aubin CE, Lacroix M, Lafon Y, Labelle H (2004) Biomechanical modelling of orthotic treatment of the scoliotic spine including a detailed representation of the bracetorso interface. Medical and Biological Engineering and Computing 42:339-344.

23. Perie D, Aubin CE, Petit Y, Labelle H, Dansereau J (2004) Personalized biomechanical simulations of orthotic treatment in idiopathic scoliosis. Clinical Biomechanics 19:190195.

24. Clin J, Aubin CE, Labelle H (2007) Virtual prototyping of a brace design for the correction of scoliotic deformities. Medical and Biological Engineering and Computing 45:467-473.

25. Clin J, Aubin CE, Parent S, Labelle H (2011) Biomechanical modeling of brace treatment of scoliosis: effects of gravitational loads. Medical and Biological Engineering and Computing 49:743-753.

26. Richards BS, Bernstein RM, D'Amato CR, Thompson GH (2005) Standardization of criteria for adolescent idiopathic scoliosis brace studies: SRS Committee on Bracing and Nonoperative Management. Spine 30(18):2068-2075.

27. Humbert L, de Guise JA, Aubert B, Godbout B, Skalli W (2009) 3D reconstruction of the spine from biplanar X-rays using parametric models based on transversal and longitudinal inferences. Med Eng Phys.31(6):681-687

28. Aubin CE, Dansereau J, De Guise JA, Labelle H (1996) A study of biomechanical coupling between spine and rib cage in the treatment by orthosis of scoliosis. Ann Chir 50:641-650.

29. Howard A, Wright JG, Hedden D (1998) A comparative study of TLSO, Charleston and Milwaukee braces for idiopathic scoliosis. Spine 23:2404-2411.

30. Zhang M, Mak A (1999) In vivo friction properties of human skin. Prosthetics and Orthotics International 23:135-141.

31. Morrissy RT, Goldsmith GS, Hall EC, Kehl D, Cowie GH (1990) Measurement of the Cobb angle on radiographs of patients who have scoliosis. Evaluation of intrinsic error. J Bone Joint Surg Am 72(3):320-327 
32. Carman DL, Browne RH, Birch JG (1990) Measurement of scoliosis and kyphosis radiographs. Intraobserver and interobserver variation. J Bone Joint Surg Am 72(3):328333

33. Morton A, Riddle R, Buchanan R, Katz D, Birch J (2008) Accuracy in the prediction and estimation of adherence to bracewear before and during treatment of adolescent idiopathic scoliosis. J Pediatr Orthop 28(3):336-41

34. Donzelli S, Zaina F, Negrini S (2015) Compliance monitor for scoliosis braces in clinical practice. J Child Orthop [Epub ahead of print]

35. Weinstein SL, Dolan LA, Wright JG, Dobbs MB (2013) Effects of bracing in adolescents with idiopathic scoliosis. N Engl J Med 369(16):1512-21

36. Katz DE, Durrani AA (2001) Factors that influence outcome in bracing large curves in patients with adolescent idiopathic scoliosis. Spine (Phila Pa 1976) 26(21):2354-61.

37. Sattout A, Clin J, Cobetto N, Labelle H, Aubin CE (20165 Biomechanical Assessment of Providence Nighttime Brace for the Treatment of Adolescent Idiopathic Scoliosis. Spine Deformity (accepted Dec 24, 2015)

38. Lebel DE, Al-Aubaidi Z, Shin EJ, Howard A, Zeller R (2013) Three dimensional analysis of brace biomechanical efficacy for patients with AIS. European Spine Journal 22(11) :2445-8

39. Weiss HR, Kleban A (2015) Development of CAD/CAM Based Brace Models for the Treatment of Patients with Scoliosis-Classification Based Approach versus Finite Element Modelling. Asian Spine Journal 9(5):661-7

40. Clin J, Aubin CE, Parent S, Labelle H (2010) A biomechanical study of the Charleston brace for the treatment of scoliosis Spine (Phila Pa 1976). 35(19):E940-7. 
Table 1 - Patient data at Initial Visit

Test Group

(NewBrace)

(19 females, 2 males)
Control Group

(CtrlBrace)

(16 females, 3 males)

\section{Statistical \\ comparison}

between

groups
Statistical test for

normality for each

group

\begin{tabular}{|c|c|c|c|c|c|c|c|c|}
\hline & & Mean & SD & & Mean & SD & Paired t-test* & Shapiro-Wilk** \\
\hline \multirow{2}{*}{\multicolumn{2}{|c|}{ Age (yrs) }} & 13.2 & 1.4 & & 13.0 & 1.3 & $\mathrm{p}=0.67$ & NewBrace, $p=0.99$ \\
\hline & & & & & & & & CtrlBrace, $p=0.76$ \\
\hline Risser & & 0.7 & 0.8 & & 0.5 & 0.7 & $p=0.36$ & \\
\hline \multirow{2}{*}{\multicolumn{2}{|c|}{ Weight (kg) }} & 41.2 & 20.9 & & 44.8 & 22.5 & $\mathrm{p}=0.42$ & NewBrace, $p=0.22$ \\
\hline & & & & & & & & CtrlBrace, $p=0.31$ \\
\hline \multirow{2}{*}{\multicolumn{2}{|c|}{ Height (cm) }} & 146.1 & 72.8 & & 154.7 & 79.7 & $\mathrm{p}=0.96$ & NewBrace, $p=0.20$ \\
\hline & & & & & & & & CtrlBrace, $\mathrm{p}=0.20$ \\
\hline MT Cobb & $\mathrm{n}=17$ & 33.2 & 6.9 & $\mathrm{n}=19$ & 27.3 & 5.1 & $\mathrm{p}=0.06$ & NewBrace, $p=0.29$ \\
\hline Angle & & & & & & & & CtrlBrace, $\mathrm{p}=0.51$ \\
\hline L Cobb & $\mathrm{n}=15$ & 27.5 & 7.3 & $\mathrm{n}=14$ & 26.3 & 7.5 & $\mathrm{p}=0.53$ & NewBrace, $p=0.31$ \\
\hline Angle & & & & & & & & CtrlBrace, $\mathrm{p}=0.66$ \\
\hline T4-T12 & & 25.5 & 10.8 & & 25.6 & 14.3 & $\mathrm{p}=0.84$ & NewBrace, $p=0.94$ \\
\hline Kyphosis & & & & & & & & CtrlBrace, $p=0.79$ \\
\hline Maximal & & 32.9 & 8.8 & & 33.6 & 14.0 & $p=0.75$ & NewBrace, $p=0.93$ \\
\hline Kyphosis & & & & & & & & CtrlBrace, $p=0.97$ \\
\hline L1-S1 & & 53.1 & 11.5 & & 54.3 & 11.6 & $\mathrm{p}=0.89$ & NewBrace, $p=0.52$ \\
\hline Lordosis & & & & & & & & CtrlBrace, $\mathrm{p}=0.80$ \\
\hline
\end{tabular}

*Statistically significantly different for $\mathbf{p}<\mathbf{0 . 0 5}$

$* *$ Normal distribution for $\mathbf{p}>\mathbf{0 . 0 5}$ 
Table 2 - In-brace results for Cobb, kyphosis and lordosis angles

Test Group (NewBrace)

Control Group (CtrlBrace)

\begin{tabular}{lcccccc}
\hline & Mean & SD & $\begin{array}{c}\text { Shapiro-Wilk } \\
\text { normality test }\end{array}$ & Mean & SD & $\begin{array}{c}\text { Shapiro-Wilk } \\
\text { normality test * }\end{array}$ \\
\hline $\begin{array}{l}\text { MT Cobb Angle reduction } \\
\text { (degrees) }\end{array}$ & 13.8 & 5.4 & $\mathrm{p}=0.05$ & 8.2 & 5.4 & $\mathrm{p}=0.91$ \\
\hline MT Cobb Angle reduction (\%) & 43 & 15 & $\mathrm{p}=0.11$ & 29 & 21 & $\mathrm{p}=0.38$ \\
& & & & & & \\
\hline $\begin{array}{l}\text { L Cobb Angle reduction } \\
\text { (degrees) }\end{array}$ & 12.9 & 5.9 & $\mathrm{p}=0.52$ & 10.6 & 4.9 & $\mathrm{p}=0.04$ \\
\hline L Cobb Angle reduction (\%) & 46 & 18 & $\mathrm{p}=0.26$ & 40 & 12 & $\mathrm{p}=0.86$ \\
\hline T4-T12 Kyphosis & & & & & & \\
& 22.8 & 9.8 & $\mathrm{p}=0.30$ & 19.5 & 10.5 & $\mathrm{p}=0.19$ \\
\hline Maximal Kyphosis & 30.1 & 9.9 & $\mathrm{p}=0.17$ & 27.1 & 8.5 & $\mathrm{p}=0.49$ \\
\hline L1-S1 Lordosis & 45.7 & 9.3 & $\mathrm{p}=0.86$ & 45.8 & 9.9 & $\mathrm{p}=0.73$ \\
\end{tabular}

*Normal distribution for $\mathbf{p}>\mathbf{0 . 0 5}$ 


\section{Figures}
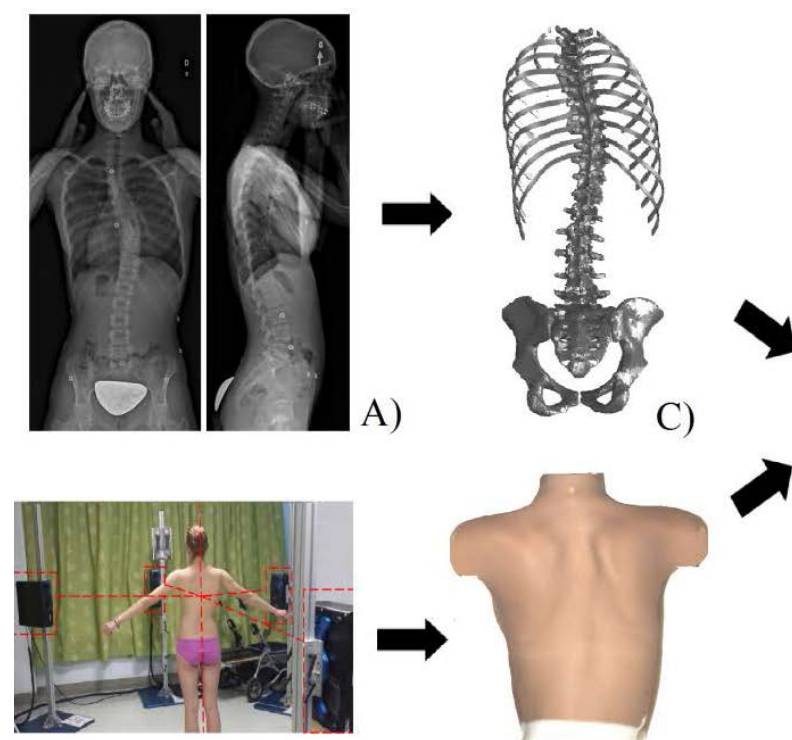

B)

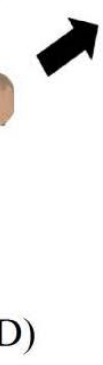

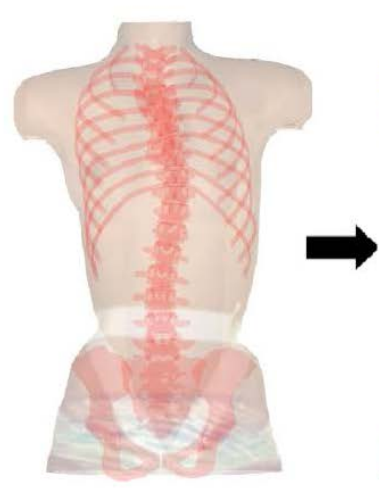

E)

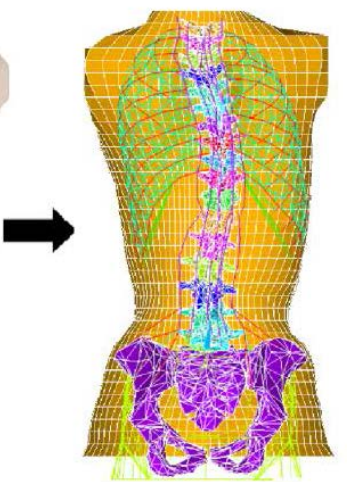

F )

Figure 1-A) Acquisition of the calibrated bi-planar radiographs; B) Surface topography acquisition; C) 3D reconstruction of the spine, rib cage and pelvis; D) Torso 3D geometry E) Geometric registration F) Finite element model of the trunk: vertebrae, intervertebral discs, ribs, sternum, costal cartilages, ligaments and soft external tissues 


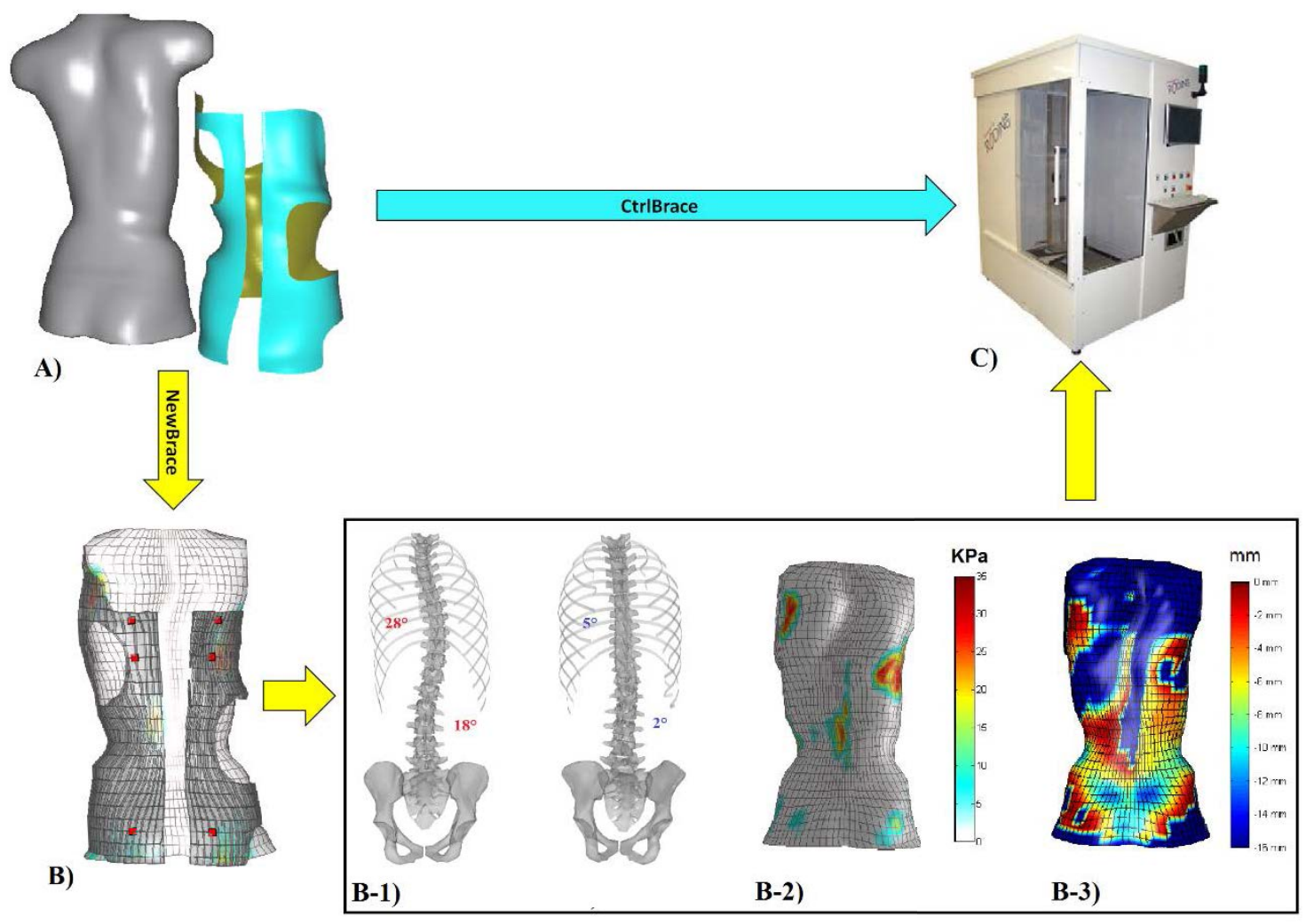

Figure 2-A) CtrlBrace or Newbrace design using the CAD software; B) Simulation of the Newbrace installation; B1) Simulation of the spine correction; B2) Simulation of the applied pressures; B3) Simulation of the distance between the brace shell and the patient's skin (the blue color represents the material in contact with the patient's skin and the green, yellow, orange and red colors represent the brace material situated at more than $6 \mathrm{~mm}$ of the patient's skin); C) Brace fabrication using a numerically controlled carver 


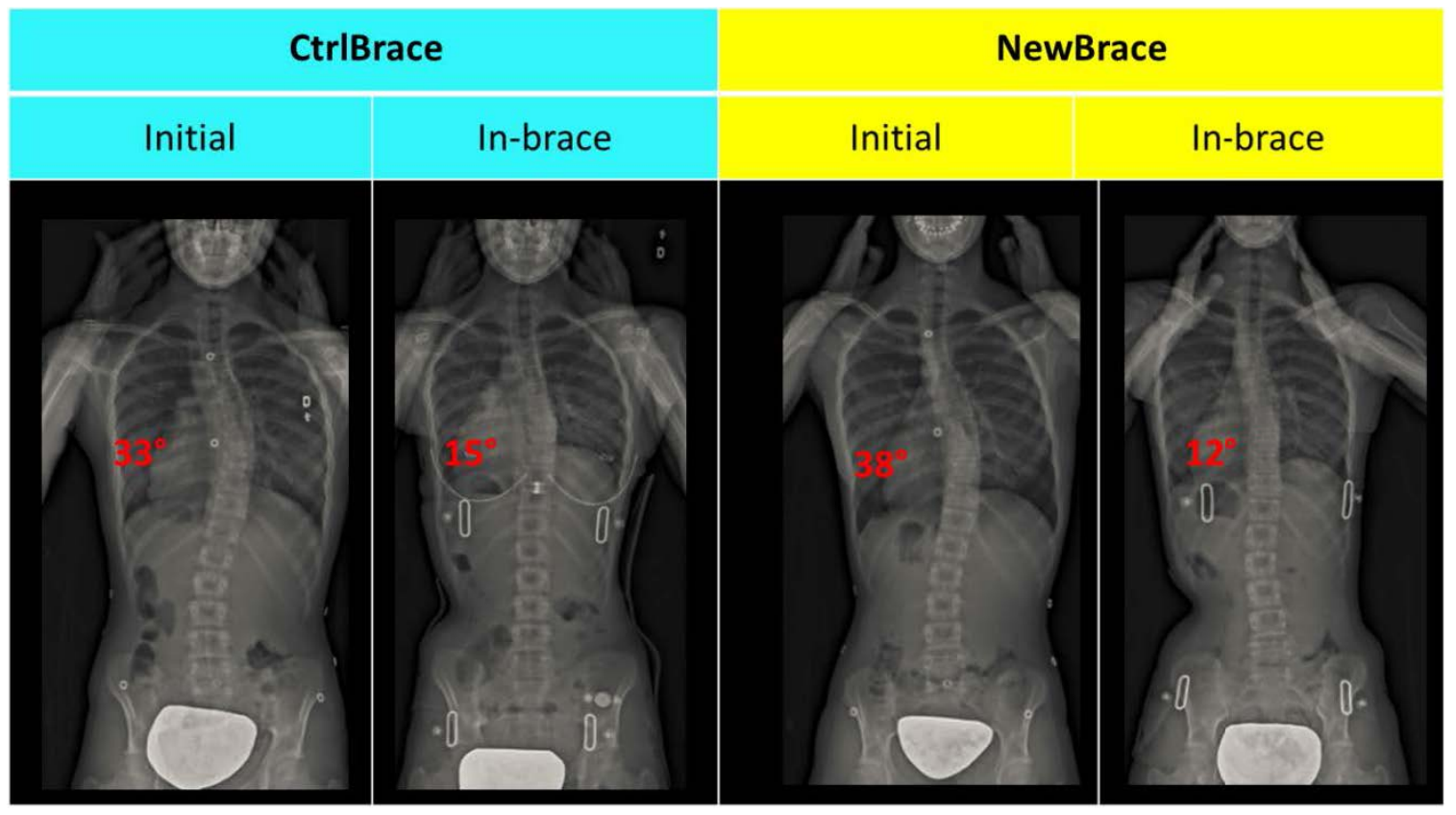

Figure 3 - PA Radiographic results for two typical patients: out of brace initial curve, with the CtrlBrace or with the NewBrace 